\title{
Motivação e Resistência ao Uso da Tecnologia da Informação: um Estudo entre Gerentes
}

\author{
Donaldo de Souza Dias
}

\section{RESUMO}

Este trabalho analisa os aspectos motivadores e a resistência ao uso da tecnologia da informação por parte dos gerentes brasileiros. Foram coletados dados junto a gerentes pertencentes a 43 empresas com o objetivo de testar os fatores intrínsecos e extrínsecos que influenciam o uso desta tecnologia nos seus ambientes de trabalho. Investigamos três motivadores: utilidade, facilidade de uso e prazer. Os resultados mostram que estes três motivadores são interrelacionados e que o aspecto lúdico existente no uso de computadores é fator importante na avaliação destes equipamentos como úteis para os gerentes em suas atividades diárias. Por outro lado, a resistência pessoal ao uso da tecnologia da informação, em particular microcomputadores, aumenta a percepção destes equipamentos como instrumentos difíceis de serem usados e, indiretamente, diminui a percepção de sua utilidade. À luz destes resultados, são discutidas oportunidades e ações que cumpre sejam tomadas para melhor capacitar as empresas brasileiras no uso da moderna tecnologia da informação.

Palavras-chaves: tecnologia da informação; interação homem-computador; motivação intrínseca; resistência à tecnologia; utilidade de microcomputadores.

\begin{abstract}
This paper analyses the motivational aspects and resistance to the use of information technology by Brazilian managers. Data were gathered from managers working for 43 companies to test the intrinsic and extrinsic factors that influence the use of this technology in their workplace. Three motivating factors were studied: usefulness, easy of use and enjoyment. The results show that these motivators are interrelated and that enjoyment is an important factor for evaluating this technology as a useful one for managers' daily activities. On the other hand, personal resistance to information technology, particularly microcomputers, increases the perception of this technology as a difficult one to be used and, indirectly, lessens the perception of its usefulness. Based on these results, we discuss opportunities and actions to be taken to capacitate Brazilian companies to do a better use of modern information technology.
\end{abstract}

Key words: information technology; human computer interaction; intrinsic motivation; resistance to technology; microcomputer usefulness. 


\section{INTRODUÇÃO}

A implantação da tecnologia da informação em uma organização é uma intervenção feita na organização visando a mudar o seu estado, com o intuito de aumentar a sua eficácia e eficiência. A utilização de microcomputadores para ajudar os indivíduos nas suas tarefas e tomada de decisão é uma das ações mais importantes que empreendemos, quando implementamos esta tecnologia efetivamente; entretanto o resultado destas intervenções, nem sempre coroadas do êxito esperado, e muitas vezes geradoras de dificuldades no que se refere à participação das pessoas no processo, nos levam freqüentemente a perguntar:

\section{- Que é que motiva os indivíduos a usar a tecnologia da informação nas organizações?}

\section{. Por que certos indivíduos resistem ao uso desta tecnologia?}

Embora seja reconhecido que os microcomputadores trazem benefícios para os indivíduos e as organizações, algumas pessoas resistem ao seu uso no ambiente de trabalho e na vida privada. Um fato bem conhecido é a dificuldade que certas pessoas têm em lidar com os caixas automáticos bancários, sendo um exemplo extremo desta situação o estado de angústia que se apossa de certos aposentados ao terem de retirar mensalmente suas pensões, utilizando os cartões magnéticos fornecidos pelo INSS. Todos nós conhecemos casos em nossos relacionamentos que exemplificam esta realidade.

De acordo com Igbaria, Parasuraman e Baroudi (1996), “o uso atual de microcomputadores por profissionais e gerentes não têm correspondido às expectativas das empresas". Isto resulta em que o benefício potencial destas tecnologias não vem sendo completamente realizado. Tal situação tem levado, freqüentemente, à subutilização dos investimentos feitos em tecnologia da informação e à pressão sobre os recursos humanos das empresas para a utilização crescente de uma tecnologia em constante mutação que, muitas vezes, não é bem entendida e até os seus usuários lhe resistem.

Pesquisas teóricas e empíricas têm tentado entender os motivadores mais importantes para a implantação e uso bem sucedido da tecnologia da informação nos ambientes de trabalho (Davis, 1989; Moore e Benbasat, 1991; Adams, Nelson e Tood, 1992; Igbaria, Iivari e Maragahh, 1995; Igbaria, Parasuraman e Baroudi, 1996). Existe um consenso de que as pessoas envidam esforços para realizar 
tarefas que lhes são agradáveis e oferecem recompensas, e que o uso de qualquer tecnologia é determinado por motivação tanto extrínseca quanto intrínseca (Deci, 1975; Gill, 1996; Igbaria, Parasuraman e Baroudi, 1996). Entretanto Igbaria, Iivani e Maragahh (1995) afirmam que "temos estudado menos as razões intrínsecas que levam os indivíduos a aceitar a tecnologia da informação do que os motivadores extrínsecos que levam à sua utilização". É de se notar que esta situação é particularmente grave em nosso país, onde se chega a considerar como falta de capacitação geral para o trabalho qualquer dificuldade ou resistência ao uso de microcomputadores.

Nesta pesquisa enfocamos os três principais motivadores para o uso de computadores estudados na literatura, conforme percebidos pelos usuários: utilidade (Moore e Benbasat, 1991; Adams, Nelson e Todd, 1992; Igbaria, Iivari e Maragahh, 1995), facilidade de uso (Davis, 1989; Igbaria, Iivani e Maragahh, 1995) e prazer (Igbaria, Iivari e Maragahh, 1995; Igbaria, Parasuraman e Baroudi, 1996). Pretendemos descobrir, em um primeiro momento:

- como os gerentes brasileiros se sentem relativamente ao uso da tecnologia dos computadores em seu ambiente de trabalho;

. como o prazer em usar computadores e a percepção da facilidade de seu uso afetam o reconhecimento da utilidade dos computadores.

Buscamos encontrar oportunidades para agir de acordo com este conhecimento visando a aumentar a qualidade do uso da tecnologia da informação em nossas organizações. Neste estudo nós enfatizamos os relacionamentos entre os motivadores estudados e a relação destes motivadores com a resistência ao uso de computadores no ambiente de trabalho. $\mathrm{O}$ impacto dos motivadores e da resistência ao uso no volume e sofisticação da utilização efetuada, ou mesmo na decisão de adotar esta tecnologia, foi considerado estar fora do escopo do nosso trabalho. Nosso estudo endereça-se especificamente às percepções dos gerentes brasileiros, no que tange aos fatores motivadores e aos bloqueios relacionados ao uso da tecnologia da informação em geral e, em particular, à tecnologia dos microcomputadores.

Neste trabalho propomos, inicialmente, um modelo que relaciona os fatores motivadores e a resistência ao uso de computadores, com base em conhecimentos teóricos da área de psicologia. Em seguida, detalhamos as variáveis e a metodologia utilizada na pesquisa. Após a análise dos resultados obtidos, discutimos a existência de fatores que poderiam ampliar o escopo de estudos futuros sobre o tema e apresentamos nossas conclusões sobre o estudo realizado. Os resultados obtidos em nossa pesquisa ressaltam a pertinência e a validade do modelo proposto. 


\section{O Modelo da Pesquisa}

As relações entre os motivadores e a resistência ao uso de microcomputadores foram analisadas por meio do modelo proposto na Figura 1. Ele está baseado na precedência natural de estímulos intrínsecos sobre estímulos extrínsecos no comportamento do ser humano, conforme proposto pela teoria da psicanálise (Freud, 1974, 1976a, 1976c).

Sabemos que uma análise baseada em relacionamentos não pode ser utilizada diretamente para justificar causalidade, uma vez que correlações medem apenas o grau em que variáveis se comportam de maneira semelhante; entretanto estamos usando um modelo path analysis com base na visão de Blalock (1972), segundo a qual "o objetivo básico de qualquer ciência é estabelecer relações de causalidade e, uma vez que a teoria é capaz de mostrar uma conexão lógica entre duas variáveis, poderemos fazer o exercício intelectual de utilizar uma interpretação causal". Prazer e resistência ao uso são os estímulos intrínsecos básicos que, em nosso modelo, influenciarão a percepção dos usuários com relação à facilidade de uso e à utilidade dos computadores em seus ambientes de trabalho.

Figura 1: Modelo Motivacional do Uso da Tecnologia da Informação

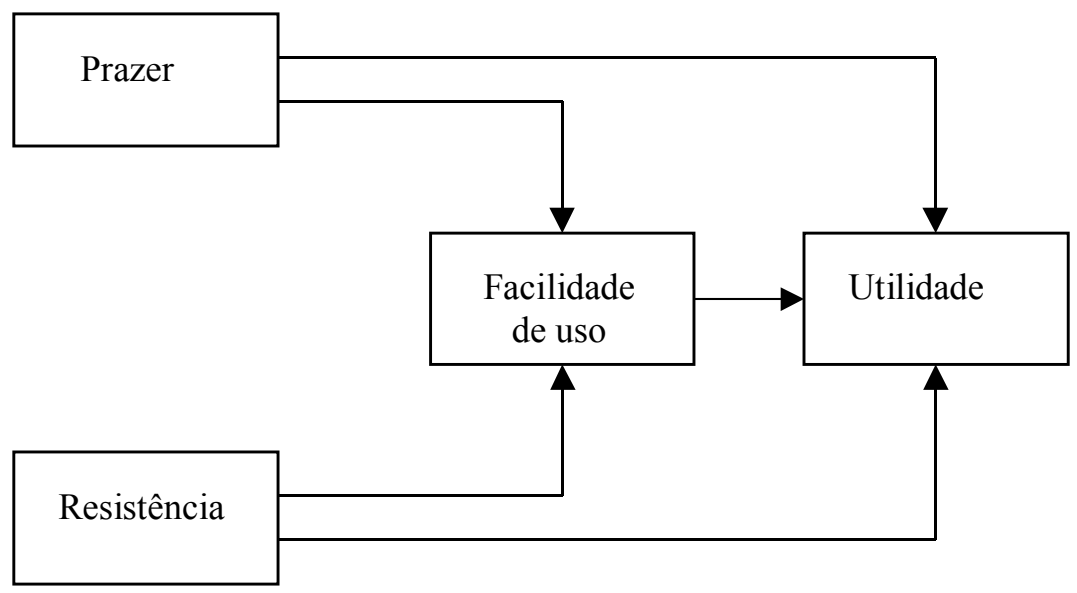




\section{VARIÁVEIS}

Utilizamos o conceito de utilidade percebida com relação ao uso de microcomputadores conforme definido por Davis (1986), no desenvolvimento do seu technology acceptance model: "grau em que um indivíduo acredita que utilizar um sistema particular melhoraria o seu desempenho no trabalho". Foram utilizadas sentenças para capturar a utilidade do uso de computadores, percebida pelos gerentes no seu ambiente de trabalho, que consideraram estes aspectos:

. qualidade do trabalho;

. rapidez na execução das tarefas;

. melhoria na produtividade;

. utilidade dos computadores para o trabalho em geral.

O conceito de facilidade percebida pelo usuário para o uso de microcomputadores foi definido por Davis (1986) no technology acceptance model: "grau em que um indivíduo acredita que utilizar um sistema particular seja livre de esforço físico ou mental". Foram usadas sentenças para capturar a facilidade percebida pelos gerentes relativamente ao uso de computadores em seu ambiente de trabalho, considerando:

. facilidade de uso da tecnologia em si;

. facilidade do uso de computadores na execução de tarefas;

. facilidade de uso para o apoio ao trabalho gerencial como um todo.

Utilizamos o conceito de prazer percebido pelo usuário com o uso de microcomputadores conforme foi definido por Davis, Bagozzi e Warshaw (1992): "grau em que a atividade de usar o computador é percebida como prazerosa em si mesma, independentemente de qualquer conseqüência de desempenho que possa ser antecipada". Frases para capturar o prazer percebido pelos gerentes relativamente ao uso de computadores em seu ambiente de trabalho consideraram os seguintes sentimentos:

. excitação;

envolvimento;

. diversão.

Alguns indivíduos criam bloqueios pessoais ao uso da tecnologia em geral (Pirsig, 1984) e, em particular, à tecnologia de computadores (Raub, 1981; 
Heinssen, Glass e Knight, 1987; Igbaria e Parasuraman, 1989; Martocchio, 1994). Eles resistem ao uso da tecnologia, experimentam angústia, um estado particular de ansiedade, quando têm de lidar com computadores. Freud (1976b) definiu que "a angústia resulta de um perigo ou uma ameaça de perigo, [...] como sentimento, ela tem uma característica claramente desagradável". A resistência ao uso de computadores pelos gerentes em seu ambiente de trabalho foi capturada em frases que apontavam:

- a angústia experimentada para a execução de tarefas que necessitassem o uso de computadores;

. o nível de motivação com relação ao uso desta tecnologia;

. quão agradável era percebido o uso dos computadores pelos gerentes.

\section{Metodologia}

Foi realizado um levantamento com 55 gerentes participantes do MBA/ Executivo do Instituto de Ensino e Pesquisa em Pós-Graduação da Universidade Federal do Rio de Janeiro (COPPEAD/UFRJ). Os respondentes trabalhavam em 43 organizações, que variavam de pequenas empresas a grandes corporações, localizadas no Estado do Rio de Janeiro. As empresas eram: 23\% multinacionais; $58 \%$ de capital privado, brasileiro; e $19 \%$ do Governo. A idade média dos respondentes foi 36 anos, com uma média de 11 anos de experiência profissional. Todos os participantes da pesquisa possuíam curso superior completo.

O questionário utilizado tinha 15 afirmativas, apresentadas de forma aleatória, cobrindo as 4 variáveis estudadas na pesquisa, usando escala Likert de 7 pontos, variando de (1) discordo totalmente a (7) concordo plenamente, conforme se apresenta no Anexo 1. As variáveis obtidas, que utilizaram 13 das 15 afirmativas do questionário, alcançaram bom coeficiente de confiabilidade interna (alfa de Crombach), conforme podemos ver a seguir:

. utilidade: $\alpha=0,89$;

. facilidade de uso: $\alpha=0,90$;

. prazer: $\alpha=0,68$;

. resistência: $\alpha=0,74$.

O modelo path analysis foi testado utilizando-se o procedimento regressão múltipla, do SPSS para o Windows. Foi tomado cuidado para minimizar as 
violações das suposições que baseiam o path analysis (Billings e Wroten apud Igbaria e Parasuraman, 1989; James apud Igbaria e Parasuraman, 1989). O exame da correlação entre as variáveis independentes, prazer no uso e resistência ao uso de computadores, não revelou multicolinearidade, isto é, seu coeficiente de correlação foi menor que 0,80 . Na verdade, estas duas variáveis apresentaram coeficiente de correlação negativo e efeitos opostos sobre as variáveis dependentes, conforme poderemos ver na análise dos resultados da pesquisa apresentados a seguir.

Visando a entender melhor os resultados do levantamento e gerar recomendações para aumentar o nível de motivação e satisfação com o uso da tecnologia da informação em nosso país, realizamos um estudo qualitativo, independente, com 22 participantes do MBA/Executivo que não participaram de nossa pesquisa quantitativa e que possuíam perfil semelhante àquele dos participantes do levantamento. Eles foram divididos em seis grupos, sendo pedido a cada grupo isto:

- discutir as suas experiências no uso da tecnologia da informação em suas empresas, levando em consideração: resistências pessoais ao uso de computadores, situações em que esta tecnologia é utilizada de maneira compulsiva pelos funcionários, motivadores para o uso de computadores para a execução de tarefas e cultura organizacional;

propor ações que achassem que deveriam ser tomadas para a implantação da tecnologia da informação em seus ambientes de trabalho e que pudessem levar a um uso mais eficaz desta tecnologia.

\section{Resultados}

A Tabela 1 apresenta o valor médio das respostas para as afirmativas do questionário e a média obtida para as variáveis da pesquisa. Os gerentes entrevistados se mostraram muito motivados pela utilidade dos computadores (média=6,29; d.p. $=0,86$ ). Os maiores motivadores para usar a tecnologia de computadores no trabalho foram:

. são úteis para o trabalho (média $=6,44 ;$ d.p. $=0,90$ );

. aumentam a qualidade do trabalho (média $=6,33$; d.p. $=0,98$ );

agilizam a execução das tarefas (média $=6,25 ;$ d.p. $=1,00$ ); e

melhoram a produtividade (média $=6,13 ;$ d.p. $=1,07$ ). 
Os baixos valores encontrados para o desvio padrão das afirmativas relativas à utilidade dos computadores mostram alto grau de concordância dos entrevistados com relação à utilidade dos computadores como ferramenta de apoio para o seu dia-a-dia no trabalho.

\section{Tabela 1: Motivação e Resistência}

\begin{tabular}{|lcc|}
\hline \multicolumn{2}{|c|}{ Descrição } & \multicolumn{2}{c|}{ Média } \\
Utilida de & Afirmativa & Variável \\
Aumenta a qualidade do trabalho & 6,33 & $\mathbf{6 , 2 9}$ \\
Agiliza a execução das tarefas & 6,25 & \\
Melhora a produtividade & 6,13 & \\
Úteis para o trabalho & 6,44 & \\
Facilidade de us o & & $\mathbf{5 , 4 0}$ \\
Fácil para executar tarefas & 5,69 & \\
Fácil para realizar trabalho & 5,35 & \\
Fácil de usar & 5,15 & \\
& & $\mathbf{4 , 2 8}$ \\
$\quad$ Excitante de usar & 4,53 & \\
Esquecer o tempo quando usa & 3,75 & \\
Usar é divertido & 4,56 & \\
Prazer $\quad$ & $\mathbf{2 , 2 6}$ \\
Angústia ao usar & 1,93 & \\
Desmotivado em utilizar & 2,45 & \\
Desagradável de usar & 2,41 & \\
\hline
\end{tabular}

Os gerentes entrevistados, em geral, declararam achar fácil utilizar computadores (média $=5,40 ;$ d.p. $=1,36$ ) e sentir prazer em utilizá-los (média=4,28; d.p.=1,41). A maior variância de facilidade de uso e prazer em usar o computador mostra que há menor unanimidade quanto ao poder motivador destes fatores para os gerentes brasileiros. Encontramos baixo grau de resistência (média=2,26; d.p. $=1,10)$ ao uso da tecnologia da informação. Este é um resultado muito positivo e mostra como o nosso corpo gerencial está preparado para as mudanças que estão acontecendo na maneira de operar e gerenciar as nossas empresas.

Nossos resultados confirmaram o encontrado por Igbaria, Parasuraman e Baroudi (1996), em estudo realizado com profissionais e gerentes de empresas americanas, em que a utilidade dos computadores foi considerada como fator mais importante do que os aspectos lúdicos e de pressão social para a utilização destes equipamentos. Confirmaram, também, o resultado do estudo realizado por Igbaria, Iivari e Maragahh (1995) com profissionais e gerentes de empresas finlandesas, que declararam usar computadores, principalmente, por perceberem 
que eram ferramentas úteis para melhorar a sua atuação no trabalho e, secundariamente, por achar agradável usá-los.

Os coeficientes de correlação entre as quatro variáveis de nosso modelo se apresentaram significantes, como pode ser visto na Tabela 2. Estes resultados mostram, em uma primeira análise, o forte coeficiente negativo de correlação entre a facilidade de uso de computadores e a resistência ao seu uso $(-0,7519$, $\mathrm{p} \leq 0,001)$.

Tabela 2: Matriz de Coeficientes de Correlação

\begin{tabular}{|lccc|}
\hline & Utilidade & Facilidade uso & Prazer \\
Facilidade uso & $0,4576^{* * *}$ & & \\
Prazer & $0,4534 * * *$ & $0,4433^{* * *}$ & \\
Resis tência & $-0,3539^{* *}$ & $-0,7519 * * *$ & $-0,4693 * * *$ \\
$* * \mathrm{p} \leq 0,01 ; * * * \mathrm{p} \leq 0,001$ & & & \\
\hline
\end{tabular}

Os resultados da regressão múltipla e os coeficientes beta representando os efeitos diretos das diversas variáveis apresentam-se na Figura 2. Ela documenta a rede dos relacionamentos causais significantes entre as variáveis da pesquisa. Quanto mais o gerente tem resistência pessoal à tecnologia de computadores, mais difícil ele acha usá-los. Encontramos um coeficiente beta=-0,70 com grau de significância $\mathrm{p} \leq 0,001$ para esta relação. Por outro lado, a percepção da utilidade dos computadores é diretamente influenciada pelo prazer em usá-los (beta $=0,32$; $\mathrm{p} \leq 0,05)$ e pela percepção de que eles são fáceis de usar (beta $=0,39 ; \mathrm{p} \leq 0,05)$. A influência da resistência ao uso de computadores por parte dos gerentes sobre a percepção de sua utilidade acontece apenas indiretamente, por meio da influência negativa da resistência sobre a facilidade de uso.

Considerando-se, entretanto, a relação negativa existente entre resistência ao uso de computadores e o prazer em seu uso $(\mathrm{r}=-0,4693 ; \mathrm{p} \leq 0,001)$, poderíamos afirmar que, se conseguirmos maneiras de diminuir o sentimento inconsciente de rejeição à tecnologia da informação, existente em certos gerentes, poderemos aumentar neles o prazer do uso desta tecnologia e gerar uma sensação de maior facilidade de uso. A conjugação destes dois efeitos levaria, certamente, a uma maior conscientização da utilidade da tecnologia da informação por parte destes gerentes. Esta suposição é coerente com os resultados de Martocchio (1994). Ele verificou, em experimento de campo realizado com funcionários em treinamento, que aqueles que revelaram um comportamento que buscava a aquisição de competência sentiram uma diminuição de sua ansiedade com relação ao uso de microcomputadores e um aumento do sentimento de uso eficaz destes equipamentos, quando comparados com empregados já acomodados com relação às competências que possuíam. 


\section{Figura 2: Resultados do Modelo}

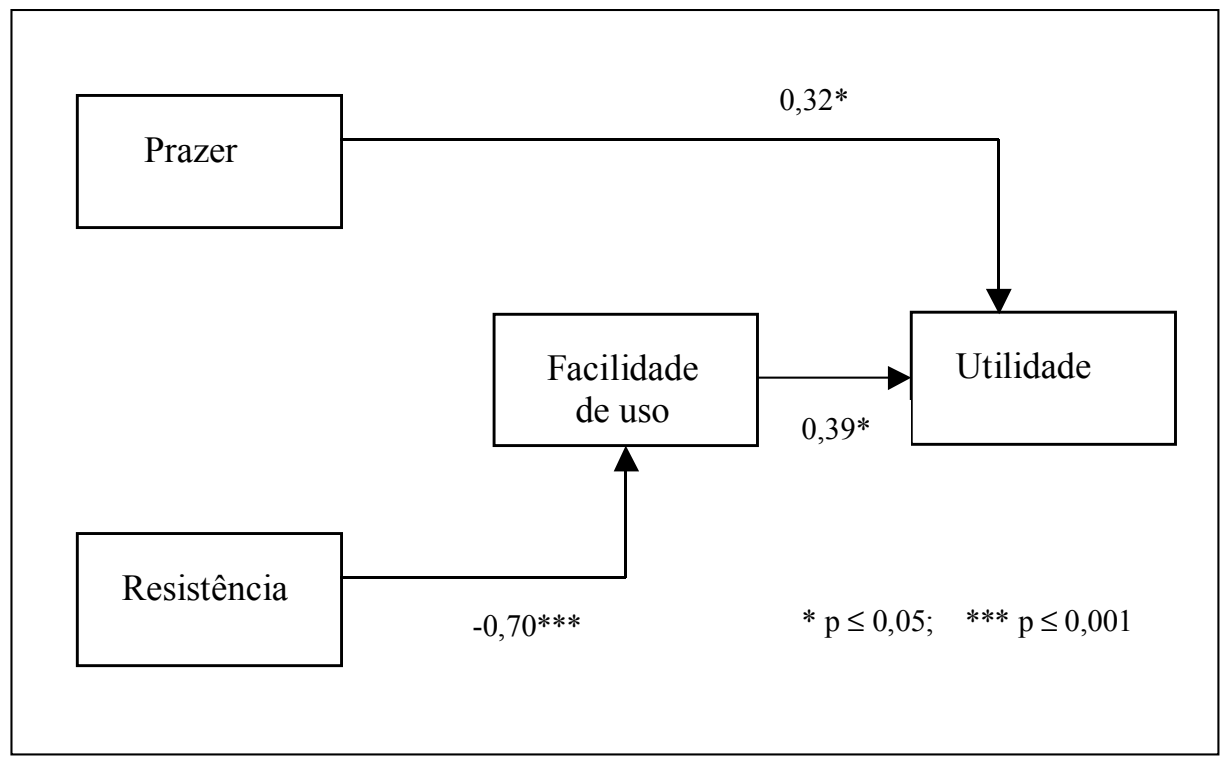

As principais ações sugeridas no estudo qualitativo, consistentes com Whyte, Bytheway e Edwards (1997), e os fatores motivacionais correspondentes por nós atribuídos aparecem na Tabela 3. Como podemos verificar pelo exposto naquela tabela, as ações sugeridas pelos grupos de trabalho são coerentes com os fatores motivacionais obtidos em nosso levantamento e consideram principalmente os aspectos de utilidade e facilidade de uso.

\section{Tabela 3: Ações para a Implantação Bem Sucedida da Tecnologia da Informação}

\begin{tabular}{|ll|}
\hline \multicolumn{1}{|c|}{ Ação } & Fator motivacional \\
\hline Desenvolver sistemas amigáveis & facilidade uso/prazer \\
Participação do usuário no desenvolvimento & utilidade/facilidade uso \\
Treinamento intensivo do usuário & facilidade uso \\
Vender o sistema para os usuários & utilidade \\
Acesso fácil aos microcomputadores & facilidade uso \\
Responder às necessidades dos usuários & utilidade \\
Usar tecnologia atualizada & utilidade/prazer \\
Usar tecnologia alinhada ao negócio da empresa & utilidade \\
\hline
\end{tabular}




\section{Discussão}

Em contexto de estudo tão complexo como o que foi por nós abordado, fica claro que existem outros fatores que afetam o modelo proposto, principalmente se escolhermos uma população alvo menos específica. Podemos dizer que, no caso de nosso estudo, controlamos as seguintes variáveis antecedentes do modelo:

nível de educação (todos com nível superior);

ocupação (todos gerentes operacionais de firmas importantes);

· região demográfica (todos da região do Rio de Janeiro).

Duas variáveis foram propositadamente por nós desconsideradas como antecedentes neste estudo: idade e sexo. Por um lado, havia uma baixa relação sexo feminino/masculino (cerca de $15 \%$ ) e era relativamente pequeno o intervalo das idades dos respondentes ( 28 a 44 anos - todos da mesma geração). Por outro lado, julgamos que sexo e idade costumam ser usados, freqüentemente, de maneira discriminatória no que concerne ao julgamento da capacidade de uso da tecnologia da informação. Por isto decidimos, no caso deste estudo, nos ater apenas às medidas da percepção dos gerentes relativas à sua interação com os microcomputadores.

Para estudos futuros, a serem realizados em outras populações, estas e outras variáveis antecedentes poderão ser consideradas. Em particular, sugerimos a incorporação ao modelo de variáveis antecedentes que meçam traços de personalidade, baseadas na teoria de Jung (1971), como as desenvolvidas por Myers e Briggs (apud Briggs e Myers, 1983) ou Keirsey (apud Keirsey e Bates, 1978).

\section{ConClusĀo}

A pesquisa aqui descrita oferece várias contribuições para o entendimento dos aspectos motivadores e das resistências que os gerentes brasileiros têm com relação ao uso de computadores em seu ambiente de trabalho. O estudo confirmou a existência e o interrelacionamento de três motivadores citados na literatura: utilidade, facilidade de uso e prazer. Os gerentes afirmaram que usam a tecnologia de computadores principalmente porque acham que ela aumenta a qualidade do seu trabalho, agiliza a execução das tarefas sob a sua responsabilidade e melhora a sua produtividade. $\mathrm{O}$ estudo também mostrou a importância de que os computadores sejam fáceis de usar. 
Embora os gerentes tenham mostrado menos unanimidade com relação ao prazer em usar computadores, este motivador mostrou claramente a sua importância. O prazer age como motivador intrínseco, enquanto a utilidade é claramente motivador extrínseco, algo que fazemos para atingir os objetivos que são colocados pela organização. A competitividade com que são realizados os negócios hoje, e a ênfase na obtenção de mais eficiência na operação das empresas levam a um cuidado especial com o aspecto da utilidade da tecnologia da informação nas organizações.

Os gerentes que participaram do estudo qualitativo enfatizaram que os sistemas de informação devem estar alinhados com o negócio da organização, devem ser amigáveis, fáceis de acessar, usar uma tecnologia atualizada e serem desenvolvidos com a participação dos usuários. Os sistemas devem, ainda, atender completamente às necessidades dos usuários, os quais devem ter amplo conhecimento e adequado treinamento sobre eles. As ações sugeridas por aqueles gerentes para uma implantação bem sucedida da tecnologia da informação influenciariam principalmente a utilidade e a facilidade de uso desta tecnologia nas empresas. Duas das ações propostas poderiam, segundo os gerentes, estimular o prazer com o uso da tecnologia da informação no ambiente de trabalho e diminuir a resistência ao seu uso: o desenvolvimento de sistemas amigáveis e a utilização de tecnologia atualizada. Considerando-se o papel motivador do prazer na utilização de computadores e o efeito nocivo da resistência ao seu uso, demonstrados em nossa pesquisa, deve ser motivo para indagações e estudos futuros verificar que outras ações poderiam atuar diretamente nestas variáveis, visando a aumentar a atratividade do uso de computadores pelos gerentes brasileiros.

O modelo da pesquisa mostrou que quanto mais o gerente tem resistência pessoal à tecnologia de computadores, mais difícil ele acha usá-los. Por outro lado, a percepção da utilidade dos computadores é diretamente influenciada pelo prazer em usá-los e pela percepção de que eles são fáceis de ser usados. A influência da resistência ao uso de computadores sobre a percepção de sua utilidade acontece apenas indiretamente, como influência negativa da resistência sobre a facilidade de uso. Podemos supor que, se conseguirmos diminuir o sentimento inconsciente de rejeição à tecnologia da informação existente em certos gerentes, poderemos lograr mais prazer no uso desta tecnologia e uma sensação de maior facilidade de uso. A conjugação destes dois efeitos levaria certamente a uma crescente conscientização da utilidade da tecnologia da informação por parte desses gerentes. 


\section{ANeXo 1}

Favor marcar o seu nível de concordância ou discordância com as frases a seguir, relativas ao uso de computadores em seu ambiente de trabalho, na escala de sete pontos cujos valores variam de discordo totalmente (1) a concordo plenamente (7).

- Eu m e esqueço do tem po, quando estou usando o

$D$ iscordo C oncordo com putador.

- U sar um computador agiliza a execução de m inhas tarefas.

$\begin{array}{lllllll}1 & 2 & 3 & 4 & 5 & 6 & 7\end{array}$

- Eu m e sinto motivado em realizar atividades que utilizem $\begin{array}{llllllll}1 & 2 & 3 & 4 & 5 & 6 & 7\end{array}$ computador.

- Eu acho que é fácil usar um com putador para executar $\begin{array}{llllllll}1 & 2 & 3 & 4 & 5 & 6 & 7\end{array}$ o m eu traba ho.

- o uso de computadores é divertido.

- U sar com putadores $m$ elhora $m$ inha produtividade no traba ho.

- U sarcomputador facilita a execução de m inhas tarefas. $\begin{array}{llllllll}1 & 2 & 3 & 4 & 5 & 6 & 7\end{array}$

- É excitante usar com putadores.

- U sar computadores aum enta a qualidade do m eu traba ho.

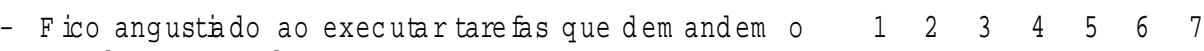
uso de computador.

- Eu acho fácil usar com putadores.

- É agradávelusar com putadores no m eu trabaho.

- Eu acho os com putadores úteis para o m eu traba ho.

- Eu acho que deveríam os usarcom putadores o máximo $\quad \begin{array}{lllllllllllll}1 & 2 & 3 & 4 & 5 & 6 & 7\end{array}$ possíve 1 .

- U sar computadores aum enta o m eu prestígi.

$\begin{array}{lllllll}1 & 2 & 3 & 4 & 5 & 6 & 7 \\ 1 & 2 & 3 & 4 & 5 & 6 & 7 \\ 1 & 2 & 3 & 4 & 5 & 6 & 7 \\ 1 & 2 & 3 & 4 & 5 & 6 & 7 \\ 1 & 2 & 3 & 4 & 5 & 6 & 7\end{array}$




\section{RefERÊnCIAS BibLIOGRÁfICAS}

ADAMS, D.;

NELSON, R.;

TODD, $\mathrm{P}$.

Perceived usefulness, easy of use, and usage of information technology : a replication. MIS Quarterly, v. 16, p. 227-247, 1992.

BILLINGS, R.;

WROTEN, S.

Use of path analysis in industrial/ organizational psychology : criticisms and suggestions. Journal of Applied Psychology, v. 63, p. 677-688, 1978.

BLALOCK, $\mathrm{H}$.

Social statistics. New York : McGraw-Hill, 1972.

BRIGGS, K.;

MYERS, I.

Myers-Briggs type indicator. Cal if ornia: Consulting Psychologists Press, 1983.

DAVIS, F.

A technology acceptance model for empirically testing new end user information systems : theory and results. [S. 1.], 1986. Thesis (Doctoral) - MIT.
Perceived usefulness, perceived ease of use, and user acceptance of computer technology. MIS Quarterly, v. 13, p. 319-340, 1989.

DAVIS, F.;

BAGOZZI, R.;

WARSHAW, P.

Extrinsic and intrinsic motivation to use computers in the workplace. Journal of Applied Social Psychology, v. 22, p. 1111-1132, 1992.

DECI, E.

Intrinsic motivation. New York: Plenum, 1975.

FREUD, S.

Obras completas. Rio de Janeiro : Imago, 1974. v. 14. p.185233.

Obras completas. Rio de Janeiro : Imago, 1976a. v. 19. p.38.

Obras completas. Rio de Janeiro : Imago, 1976b. v. 20. p. 95201.

Obras completas. Rio de Janeiro : Imago, 1976c. v. 22. p. 75102. 
GILL, T.

Expert systems usage : task change and intrinsic motivation. MIS Quarterly, p. 301-328, Sept. 1996.

HEINSSEN, R.;

GLASS, C.;

KNIGHT, L.

Assessing computer anxiety : development and validation of the computer anxiety rating scale. Computers in Human Behavior, v. 3, p. 49-59, 1987.

IGBARIA, M.;

IIVARI, J.;

MARAGAHH, $\mathrm{H}$.

Why do individuals use computer technology? A finnish case study. Information \& Management, v. 29, p. 227-238, 1995.

IGBARIA, M.;

PARASURAMAN, S.

A path analytic study of individual characteristics, computer anxiety and attitudes toward microcomputers. Journal of Management, v. 15, p. 373388, 1989.

IGBARIA, M.;

PARASURAMAN, S.;

BAROUDI, J. J.

A motivational model of microcomputer usage. Journal of Management Information Systems, v. 13, p. 127-143, 1996.
JAMES, L.

The unmeasured variables problem in path analysis. Journal of Applied Psychology, v. 65, p. 415-421, 1980.

JUNG, C.

Psychological types. In: CAMPBELL, J. (Ed.). The portable Jung. New York : Penguin Books, 1971. p. 178269.

KEIRSEY, D.;

BATES, $M$.

Please understand me. New York : Prometheus Nemesis Book, 1978.

MARTOCCHIO, J.

Effects of conceptions of ability on anxiety, self-efficacy, and learning in training. Journal of Applied Psychology, v. 79, p. 819-825, 1994.

MOORE, G.;

BENBASAT, I.

Development of an instrument to measure the perceptions of adopting an information technology innovation. Information Systems Research, v. 2, p. 192-222, 1991.

PIRSIG, R.

Zen e a arte de manutenção de motocicletas. Rio de Janeiro : Paz e Terra, 1984. 
RAUB, A.

Correlates of computer anxiety in college students. Pennsylvania, 1981. Thesis (Doctoral) University of Pennsylvania.
WHYTE, G.;

BYTHEWAY, A.;

EDWARDS, C.

Understanding user perception of information systems success. Strategic Information Systems, v. 6, p. 37-68, 1997. 